\title{
Relative dose intensity of first-line chemotherapy and overall survival in patients with advanced non-small-cell lung cancer
}

\author{
Jeffrey Crawford ${ }^{1}$ (D) Neelima Denduluri ${ }^{2} \cdot$ Debra Patt $^{3} \cdot$ Xiaolong Jiao $^{3} \cdot$ Phuong Khanh Morrow $^{4} \cdot$ Jacob Garcia $^{4}$. \\ Richard Barron ${ }^{4}$. Gary H. Lyman ${ }^{5}$
}

Received: 21 December 2018 / Accepted: 14 May 2019/Published online: 7 June 2019

(C) The Author(s) 2019

\begin{abstract}
Purpose The effects of chemotherapy dose intensity on survival in patients with advanced non-small-cell lung cancer (NSCLC) are poorly understood. We retrospectively analyzed dose delays/reduction, relative dose intensity (RDI), and the association between chemotherapy intensity and survival in advanced NSCLC.

Methods This retrospective cohort study included adults with advanced lung cancer who received first-line myelosuppressive platinum-based chemotherapy (January 2007-December 2010) in 230 US Oncology Network community practices. Dose delays $\geq 7$ days, dose reductions $\geq 15 \%$, and RDI relative to standard regimens were described. Overall survival (OS) was measured using Kaplan-Meier and Cox proportional hazard (PH) models.

Results Among 3866 patients with advanced NSCLC, 32.4\% experienced dose delays $\geq 7$ days, $50.1 \%$ experienced dose reductions $\geq 15 \%$, and $40.4 \%$ had RDI $<85 \%$. Reduced RDI was also common regardless of baseline ECOG PS (ECOG PS $\geq 2,56.2 \%$; ECOG PS 0, 33.6\%) and tumor subgroup (squamous cell carcinoma, 52.2\%; adenocarcinoma, 36.0\%). When stratified by chemotherapy intensity measures, significant OS differences were observed only for dose delays. Median (95\% CI) OS was 1.02 years $(0.96-1.12)$ for dose delays $\geq 7$ days and 0.71 years $(0.66-0.77)$ for dose delays $<7$ days. In multivariable Cox $\mathrm{PH}$ analysis, dose delays $\geq 7$ days $(\mathrm{HR}=0.71 ; 95 \% \mathrm{CI}=0.63-0.80)$ and $\mathrm{RDI} \geq 85 \%(\mathrm{HR}=1.18 ; 95 \% \mathrm{CI}=1.05-1.32)$ were significantly associated with decreased mortality.

Conclusions Dose delays, dose reductions, and reduced RDI were common, and dose delays $\geq 7$ days and high RDI were significantly associated with decreased mortality. These results can help identify potential risk factors and characterize the effect of chemotherapy dose modification strategies on mortality.
\end{abstract}

Keywords Lung cancer $\cdot$ Chemotherapy $\cdot$ Retrospective studies $\cdot$ Community health services

Jacob Garcia and Richard Barron's affiliation is where the work was conducted.

Electronic supplementary material The online version of this article (https://doi.org/10.1007/s00520-019-04875-1) contains supplementary material, which is available to authorized users.

Jeffrey Crawford

jeffrey.a.crawford@duke.edu

1 Duke University Medical Center, Trent Drive, Duke South, 25177 Morris Building, Durham, NC 27710, USA

2 Virginia Cancer Specialists, US Oncology Network, Arlington, VA, USA

3 McKesson Specialty Health, The Woodlands, TX, USA

4 Amgen Inc., Thousand Oaks, CA, USA

5 Fred Hutchinson Cancer Research Center and the University of Washington, Seattle, WA, USA

\section{Introduction}

Lung cancer is the second most common malignancy in the USA, with an annual incidence of 55.8 per 100,000 [1]. An analysis of the National Cancer Institute's Surveillance Epidemiology and End Results (SEER) program reported that in 2010, most patients with non-small-cell lung cancer (NSCLC) were in an advanced stage at diagnosis: $8 \%$ of patients were in stage $3 \mathrm{~A}$, and $66 \%$ were in stage $3 \mathrm{~B} / 4$ [2]. Approximately half of patients in stage $3 \mathrm{~B} / 4$ receive chemotherapy to slow disease progression and prolong survival $[2,3]$. Myelosuppressive chemotherapy regimens are frequently used to treat advanced NSCLC in US clinical practice [4] and typically include carboplatin, cisplatin, paclitaxel, pemetrexed, and gemcitabine [2]. Such agents are commonly associated with toxicities, typically managed with dose delays and reductions, 
in addition to prophylactic or supportive medications $[5,6]$. Platinum-based chemotherapy is particularly associated with dose-limiting toxicities that require changes in dosing [7, 8]. Neutropenic complications (e.g., febrile neutropenia) often require delays or reductions in doses of myelosuppressive chemotherapy, which contribute to reduced relative dose intensity (RDI; typically defined as the ratio of the actual delivered chemotherapy dose to the planned dose) $[9,10]$.

In the adjuvant setting for NSCLC, reduced RDI and dose reductions have been associated with decreased survival [11]. However, the impact of dose changes should be considered in the context of overall health and comorbidities. There is limited information on the relationship between RDI and survival in patients with advanced NSCLC. A retrospective analysis of elderly patients with advanced NSCLC reported that patients who received RDI $\geq 80 \%$ experienced statistically higher response rates and overall survival (OS) than patients who received RDI $<80 \%$ [12]. To better understand chemotherapy intensity and its potential association with OS, we retrospectively analyzed dose delays, dose reductions, RDI, and the association between chemotherapy intensity and OS in patients with advanced/metastatic lung, breast, or ovarian cancer treated in a large US community-based oncology network setting. Prophylactic and reactive use of colony-stimulating factor (CSF) was also explored. Here, we report results from patients with advanced NSCLC. Results from patients with breast or ovarian cancer have been reported separately [13].

\section{Materials and methods}

\section{Study design and data source}

This was a retrospective cohort study of adult patients diagnosed with advanced lung cancer. Data were extracted from the McKesson Specialty Health/US Oncology iKnowMed ${ }^{\mathrm{TM}}$ database, a web-based electronic health record (EHR) system with oncology-specific data that includes records from more than 16 million patients and 900 US oncologist-users across nearly 230 practices. Medical chart review was not routinely conducted; however, it was performed as required to clarify data obtained from the EHR. The study protocol was submitted to the institutional review board and was exempted. The dosing analysis for this study was focused on the first-line chemotherapy course (i.e., index course) for each patient.

\section{Patients}

\section{Inclusion criteria}

Included patients were $\geq 18$ years old, were diagnosed with advanced (stage 3 or 4) NSCLC, and had received first-line intravenous myelosuppressive platinum-based chemotherapy (Table 1) between January 2007 and December 2010 from oncology practices using the full EHR capacity of iKnowMed. Patients were also required to have had $\geq 4$ visits in the US Oncology Network during the study period.

\section{Exclusion criteria}

Patients were excluded if they were enrolled in clinical trials for the treatment of advanced cancer at the time of enrollment, received only non-myelosuppressive chemotherapy agents during cycle 1 , or received any oral myelosuppressive agents during the index course.

\section{Study outcomes}

Patient data were examined for the 6-month period from initiation of chemotherapy for the estimation of dose delays, dose reductions, RDI, and incidence of RDI $<85 \%$. The incidence of CSF use, prophylactic antimicrobial use, and grade 3/4 neutropenia was also examined. For estimation of OS, patients were followed up until death or the last visit date available.

As described for the part of this study that included patients with advanced breast or ovarian cancer [13], data were extracted from the iKnowMed database on the planned chemotherapy, the planned chemotherapy dose and schedule, the planned cycle length, the actual chemotherapy delivered, the actual chemotherapy dose and schedule, and the actual cycle length.

\section{Assessments}

In this analysis, only myelosuppressive chemotherapy regimens with data for $>100$ patients with NSCLC were included to ensure a sufficiently large sample size for analysis. Grade $3 / 4$ neutropenia was defined as an absolute neutrophil count (ANC) of $<1000$ cells $/ \mathrm{mm}^{3}$ [14].

Colony-stimulating factor prophylaxis was defined as CSF use by day 5 of the chemotherapy cycle. Reactive treatment was defined as CSF use after day 5 of the chemotherapy cycle. Primary prophylaxis was defined as first CSF use within the first 5 days of chemotherapy cycle 1 of the index course; secondary prophylaxis was defined as first CSF use within the first 5 days in any subsequent cycle.

Chemotherapy dose delays, dose reductions, and RDI (Supplemental Table 1) were evaluated against standard chemotherapy regimens (Table 1). Information on standard regimens was extracted from the National Comprehensive Cancer Network guidelines version 1.2012, current at the time of this analysis [15], and from published clinical literature reporting efficacy and outcome results from phase 3 clinical studies. 
Table 1 Standard chemotherapy regimens

\begin{tabular}{llll}
\hline Chemotherapy regimen & Standard dose & Cycle length, days & Number of cycles \\
\hline Carboplatin + paclitaxel & $5 \mathrm{AUC} / 175 \mathrm{mg} / \mathrm{m}^{2}$ & 21 & 4 \\
Pemetrexed + carboplatin & $500 \mathrm{mg} / \mathrm{m}^{2} / 5 \mathrm{AUC}$ & 21 & 4 \\
Bevacizumab + carboplatin + paclitaxel & $15 \mathrm{mg} / \mathrm{kg} / 5 \mathrm{AUC} / 175 \mathrm{mg} / \mathrm{m}^{2}$ & 21 & 4 \\
Pemetrexed + cisplatin & $500 \mathrm{mg} / \mathrm{m}^{2} / 75 \mathrm{mg} / \mathrm{m}^{2}$ & 21 & 4 \\
Pemetrexed + bevacizumab + carboplatin & $500 \mathrm{mg} / \mathrm{m}^{2} / 15 \mathrm{mg} / \mathrm{kg} / 5 \mathrm{AUC}$ & 21 & 4 \\
Carboplatin + gemcitabine & $5 \mathrm{AUC} / 1000 \mathrm{mg} / \mathrm{m}^{2} \times 2$ & 21 & 4 \\
\hline
\end{tabular}

$A U C$ area under the plasma drug concentration-time curve

Dose delays, dose reductions, and RDI were assessed over the first 6 months for each myelosuppressive agent that was administered as part of the standard chemotherapy regimen. Chemotherapy doses that were administered beyond 6 months were not considered in this study. For determination of dose delays and reductions, only intravenously administered myelosuppressive agents were considered. Dose delays were defined as a delay of $\geq 7$ days from the standard regimen in any given chemotherapy cycle. A dose reduction was defined as a decrease of $\geq 15 \%$ in the chemotherapy dose relative to the standard $\left(\mathrm{mg} / \mathrm{m}^{2}\right)$ regimen for $\geq 1$ myelosuppressive agent in any given cycle. Chemotherapy RDI was defined as the ratio of the delivered dose intensity to the standard dose intensity. The RDI definition for regimens containing multiple myelosuppressive agents was the mean of the individual RDI values for each myelosuppressive chemotherapy agent included in the regimen. If the chemotherapy dose or the number of cycles received within 6 months by a patient was higher than the standard dose or number of cycles, the RDI value determined for the chemotherapy course could exceed $100 \%$. RDI $<85 \%$ is considered to be a clinically important variation from standard dosing as a part of a chemotherapy regimen $[9,16]$; therefore, this value was used as the threshold to define reduced RDI. The termination date of the index course was defined as the date of the last chemotherapy administration plus the standard cycle length, the date of death, or the date of regimen change, whichever occurred first. Standard cycle length was determined for the corresponding standard chemotherapy regimen for the agent(s) in the first cycle. OS was defined as the time interval between the initiation of the first-line chemotherapy regimen and death or last follow-up.

Data were collected on eligible patients up to December 2010. Death events were defined by the Social Security Death Index and iKnowMed; patients without a death event were censored at the date of last observed visit.

\section{Statistical analyses}

Statistical analyses were conducted as previously described for this study [13]. In brief, Kaplan-Meier unadjusted survival analyses and log-rank tests were performed to compare OS between patients with NSCLC who had chemotherapy dose delays $\geq 7$ days versus $<7$ days, dose reductions $\geq 15 \%$ versus $<15 \%$, and $\mathrm{RDI}<85 \%$ versus $\geq 85 \%$.

Univariable Cox regression analyses were performed to determine the hazard ratio (HR) for each covariate. As known risk factors and based on clinical experience, the following variables were evaluated independently as survival predictors: dose delay $\geq 7$ versus $<7$ days, dose reduction $\geq 15 \%$ versus $<15 \%$, RDI $<85 \%$ versus $\geq 85 \%$, age at first visit, Eastern Cooperative Oncology Group performance status (ECOG PS) 1 or 2 versus 0 , body surface area (BSA) $>2$ versus $\leq 2 \mathrm{~m}^{2}$, baseline $\mathrm{ANC}<1000$ versus $\geq 1000$, comorbidities $\geq 1$ versus 0 , hemoglobin $<12$ versus $\geq 12 \mathrm{~g} / \mathrm{dL}$, and tumor subgroup (adenocarcinoma or other versus squamous). Multivariable Cox models included variables that were significant and marginally significant $(P<0.10)$ from the univariable analysis and were used to adjust for covariates. Univariable and multivariable models were developed as previously described for this study [13].

\section{Results}

This study included 3866 patients with advanced NSCLC who met the inclusion criteria. Patient demographics and disease characteristics are listed in Table 2. The mean (SD) age was 66.8 years $(10.0), 56.6 \%$ of patients were male, and $61.6 \%$ were $\geq 65$ years old. A total of 892 patients $(23.1 \%)$ had a BSA $>2 \mathrm{~m}^{2}$, and 1991 (51.5\%) had an ECOG PS $\geq 1$. Overall, 2088 patients (54.0\%) had adenocarcinoma and 601 (15.6\%) had squamous cell carcinoma; tumor subgroup was unknown or data were missing in 1050 patients $(27.2 \%)$. The most common chemotherapy regimens were carboplatin + paclitaxel (1733 patients, $44.8 \%)$, pemetrexed + carboplatin (789 patients, 20.4\%), and bevacizumab + carboplatin + paclitaxel (734 patients, $19.0 \%$; Supplemental Table 2). At baseline, 86 patients $(2.2 \%)$ had grade $3 / 4$ neutropenia, defined as ANC $<1000$ cells $/ \mathrm{mm}^{3}$ (Table 2). 
Table 2 Patient demographics and baseline characteristics

\begin{tabular}{ll}
\hline Characteristic $^{\mathrm{a}}$ & $\begin{array}{l}\text { Patients with NSCLC } \\
N=3866\end{array}$ \\
\hline Mean (SD) age, years & $66.8(10.0)$ \\
Age group, years & \\
$18-49$ & $184(4.8)$ \\
$50-64$ & $1299(33.6)$ \\
$65-74$ & $1465(37.9)$ \\
$\geq 75$ & $918(23.8)$ \\
Men & $2187(56.6)$ \\
BSA $>2$ m & \\
ECOG PS & $892(23.1)$ \\
0 & \\
1 & $1484(42.6)$ \\
$\geq 2$ & $1765(50.7)$ \\
Unknown & $226(6.5)$ \\
Missing & $8(0.2)$ \\
Grade 3/4 neutropenia & \\
Tumor subgroup & $383(9.9)$ \\
Adenocarcinoma & $86(2.2)$ \\
Squamous cell carcinoma & \\
Other & $2088(54.0)$ \\
Unknown & $601(15.6)$ \\
Missing & $127(3.3)$ \\
\end{tabular}

BSA body surface area, ECOG PS Eastern Cooperative Oncology Group performance status, NSCLC non-small-cell lung cancer

${ }^{\text {a }}$ Data are expressed as $n(\%)$ unless otherwise noted

${ }^{\mathrm{b}}$ Defined as absolute neutrophil count $<1000$ cells $/ \mathrm{mm}^{3}$

\section{Dose delays, dose reductions, and relative dose intensity}

Dose delays, dose reductions, and reduced RDI were common. Overall, $32.4 \%$ of patients experienced a dose delay $\geq 7$ days, $50.1 \%$ experienced a dose reduction $\geq 15 \%$, and mean (SD) RDI across all regimens was $83.9 \%$ (28.5\%), with $40.4 \%$ of patients receiving RDI $<85 \%$. Dose delays, dose reductions, and reduced RDI were common even among young patients; among patients 18 to 49 years old, $27.7 \%$ (95\% CI, 21.2\%-34.3\%) experienced a dose delay $\geq 7$ days, $44.0 \%(36.8 \%-51.3 \%)$ experienced a dose reduction $\geq 15 \%$, and $32.1 \%(25.3 \%-38.9 \%)$ experienced RDI $<85 \%$ (Supplemental Table 3). Reduced RDI was also common regardless of ECOG PS (ECOG PS $\geq 2,56.2 \%$; ECOG PS 0,33.6\%) and tumor subgroup (squamous cell carcinoma, 52.2\%; adenocarcinoma, 36.0\%; Supplemental Table 3). Mean (SD) RDI was $75.9 \%$ (34.4\%) with carboplatin + paclitaxel, $95.0 \%(14.9 \%)$ with pemetrexed + carboplatin, and $87.1 \%(28.0 \%)$ with bevacizumab + carboplatin + paclitaxel (Supplemental Table 2).

\section{Neutropenia and supportive care}

Among 3408 patients with evaluable ANC data, 24.4\% experienced grade 3/4 neutropenia. Prophylaxis with CSFs or antimicrobials was uncommon; $18.3 \%$ received primary prophylaxis with CSFs, $6.3 \%$ received secondary prophylaxis with $\mathrm{CSFs}$, and $31.0 \%$ received prophylaxis with oral antimicrobials (Table 3).

\section{Overall survival}

All 3866 patients with NSCLC were included in the OS analysis. At the time of the analysis, a total of 2674 patients $(69 \%)$ had died. When OS was stratified by measures of chemotherapy intensity (dose delays $<7$ versus $\geq 7$ days, dose reductions $<15 \%$ versus $\geq 15 \%$, and $\mathrm{RDI}<85 \%$ versus $\geq 85 \%$ ), significant differences in OS were observed only for dose delays (Fig. 1). Median (95\% CI) OS was 1.02 years $(0.96-1.12)$ for patients with dose delays $\geq 7$ days and 0.71 years $(0.66-0.77$; $\log$-rank $P<0.0001$ ) for patients with dose delays $<7$ days.

In the univariable analysis, dose delays $\geq 7$ days (HR, 0.776 [95\% CI, 0.715-0.841]) were significantly associated with a $22.4 \%$ reduction in the risk of death (Supplemental Table 4). Other measures of chemotherapy intensity (RDI and dose reductions) were not significantly associated with OS. ECOG PS 1 versus 0 (HR, 1.364 [95\% CI, 1.255-1.483]) and 2 versus 0 (HR, $1.766[1.498-2.081])$ and hemoglobin $<12$ versus $\geq 12 \mathrm{~g} / \mathrm{dL}$ (HR, 1.205 [1.111-1.308]) were significantly associated with increased risk of death, whereas adenocarcinoma versus squamous cell carcinoma tumor subgroup (HR, 0.762 [0.685-0.847]) was significantly associated with a decreased risk of death.

The multivariable models included 2848 patients with complete data for dose reductions $(\geq 7$ versus $<7$ days), ECOG PS ( 1 versus $0 ; 2$ versus 0$)$, and hemoglobin $(<12$ versus $\geq 12 \mathrm{~g} / \mathrm{dL}$ ) and 2127 patients with complete data for those parameters as well as tumor subgroup (adenocarcinoma versus squamous; other versus squamous; Table 4). After controlling for covariates (ECOG PS, hemoglobin, and tumor

Table 3 CSF and antibiotic use

Characteristic, $n(\%)$

Patients with NSCLC $N=3866$

CSF use

Primary prophylaxis ${ }^{\mathrm{a}}$

$709(18.3)$

Secondary prophylaxis ${ }^{\mathrm{b}}$

$242(6.3)$

Treatment ${ }^{\mathrm{c}}$

$636(16.5)$

Prophylactic oral antibiotics

$1200(31.0)$

CSF colony-stimulating factor, NSCLC non-small-cell lung cancer

${ }^{\mathrm{a}}$ First receipt of CSF within first 5 days of first cycle of the index course

${ }^{b}$ First receipt of CSF within first 5 days of subsequent cycles of the index course

${ }^{\mathrm{c}}$ First receipt of CSF in any given cycle after cycle day 5 

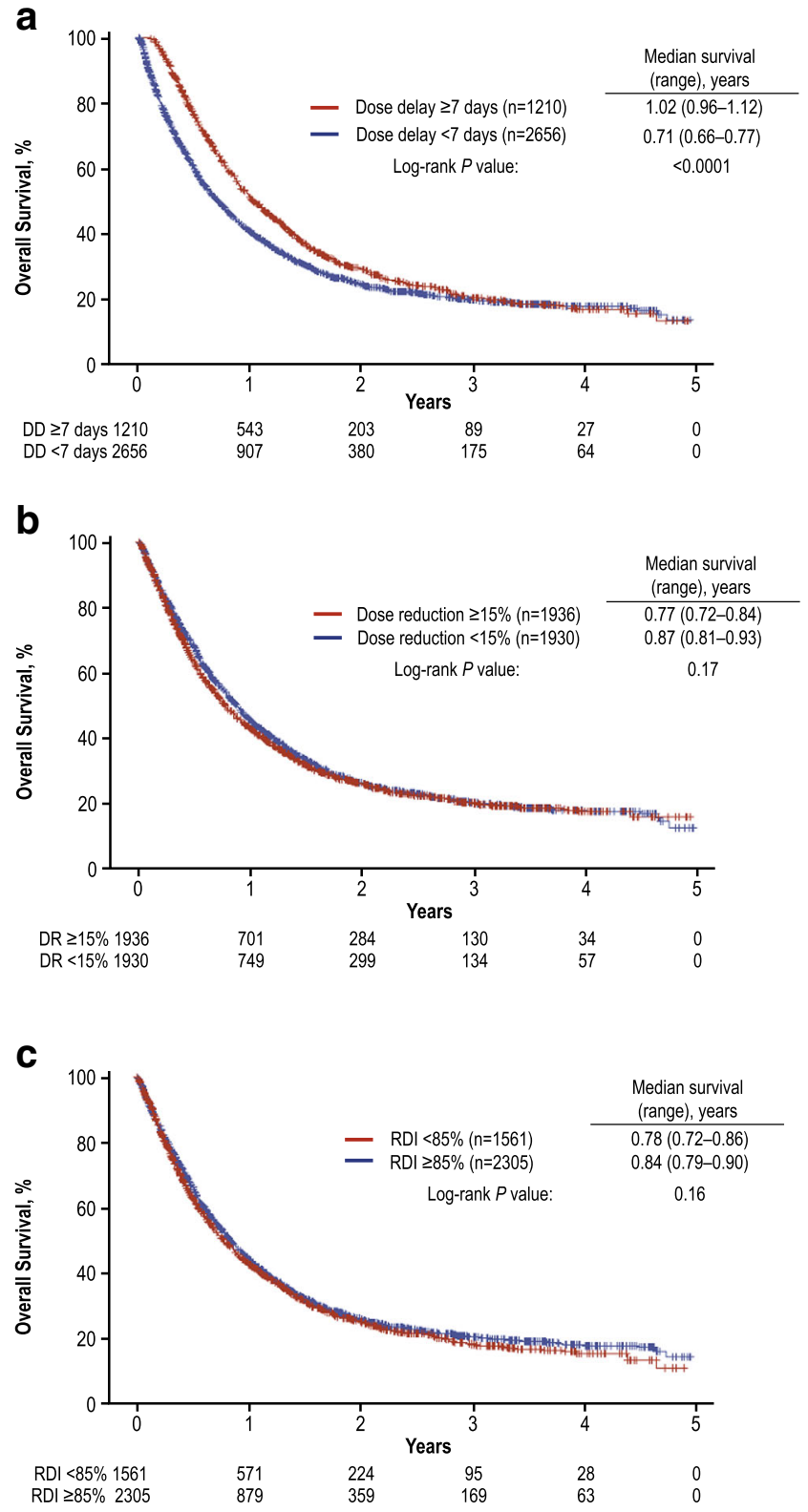

Fig. 1 Overall survival for all chemotherapy regimens by $\mathbf{a}$ dose delay, $\mathbf{b}$ dose reduction, and $\mathbf{c}$ RDI among patients with NSCLC. $D D$ dose delay, $D R$ dose reduction, $N S C L C$ non-small-cell lung cancer, $R D I$ relative dose intensity

subgroup), multivariable analysis suggested that dose delay $\geq 7$ days and increased RDI were significant predictors of longer OS (Table 4). Dose delay $\geq 7$ days was significantly associated with a $29.0 \%$ decrease in risk of death $(P<0.0001)$. RDI $\geq 85 \%$ was significantly associated with a $17.6 \%$ decrease in risk of death $(P=0.0062)$.

\section{Discussion}

Chemotherapy dosing patterns and their subsequent impact on outcomes in the real-world treatment of advanced NSCLC are
Table 4 Multivariable Cox regression analysis of OS in patients with NSCLC $^{\mathrm{a}}$

\begin{tabular}{llr}
\hline Variable & HR $(95 \% \mathrm{CI})$ & $P$ value \\
\hline RDI, $<85 \%$ vs $\geq 85 \%$ & $1.176(1.047-1.320)$ & 0.0062 \\
Dose delay, $\geq 7$ vs $<7$ days & $0.710(0.630-0.800)$ & $<0.0001$ \\
ECOG PS & $1.316(1.192-1.453)$ & $<0.0001$ \\
1 vs 0 & $1.654(1.350-2.027)$ & $<0.0001$ \\
2 vs 0 & $1.098(0.993-1.213)$ & 0.0686 \\
Hemoglobin, <12 vs $\geq 12$ g/dL & & \\
Tumor subgroup & $0.783(0.698-0.877)$ & $<0.0001$ \\
Adenocarcinoma vs squamous & $0.932(0.725-1.199)$ & 0.5855 \\
Other vs squamous & &
\end{tabular}

ECOG PS Eastern Cooperative Oncology Group performance status, $H R$ hazard ratio, NSCLC non-small-cell lung cancer, $O S$ overall survival, $R D I$ relative dose intensity

${ }^{a}$ In the overall study cohort of 3866 patients, 2674 (69\%) deaths were observed. This analysis includes 2848 patients who had complete data for dose reductions, ECOG PS, and hemoglobin and 2127 patients who had complete data for ECOG PS, hemoglobin, and tumor subgroup

not well understood. There are few chemotherapy options for patients with non-squamous subtypes of lung cancer; therefore, it is critical to optimize chemotherapy delivery for those who are treated. A recent retrospective evaluation of dose intensity for the treatment of a variety of non-metastatic cancers in the community setting (that also used the iKnowMed EHR database) found that dose delay $\geq 7$ days, dose reduction $\geq 15 \%$, and $\mathrm{RDI}<85 \%$ were frequent, including among patients treated with myelosuppressive chemotherapy regimens for NSCLC [6]. As in the earlier study [6], chemotherapy dose delays, reductions, and reduced RDI were common in patients with advanced NSCLC in this study.

Multivariable analysis suggested that dose delay $\geq 7$ days was significantly associated with a $29 \%$ decreased risk of death. The effect of dose delay $\geq 7$ days on OS was maintained out to 3 years (Fig. 1a). This suggests that the effect of dose delay on OS was not simply a reduction in toxicity, which would have had only a short-term impact on OS. One possibility is that those patients who had a dose delay of $\geq 7$ days may have had a more profound degree of cytopenias and other complications that resulted in treatment delay compared with patients who had blood cell count recovery and could proceed with chemotherapy on time but required dose reduction. In both cases, the patients had experienced dose-limiting toxicity from the treatment, and this may be a better surrogate of chemotherapy dose delivery on an individual basis than RDI. Dose reduction alone was not a significant predictor of survival in either univariable or multivariable analysis in patients with NSCLC. RDI $<85 \%$ was not significantly associated with an increased risk of death in the univariable analysis; multivariable analysis suggested that RDI $<85 \%$ was significantly associated with an $18 \%$ increased risk of death. These 
seemingly conflicting results between dose delay $\geq 7$ days and $\mathrm{RDI}<85 \%$ may have been related to multiple factors, including differences in the definitions used: RDI is a composite measure of both dose delays and reductions that compares the full delivered dose of a complete chemotherapy with the standard dose within a 6-month period for a given chemotherapy standard regimen, whereas dose delay was calculated as a delay of $\geq 7$ days in any given chemotherapy cycle from the standard regimen. Potential confounders, such as comorbidities, may also affect the results; however, they were not examined in this study. These results may also reflect physicians' comfort with dose delays in a subset of patients to refine treatment management. For example, a patient who had a dose delayed to manage hematologic toxicity, but who was subsequently able to continue with the same dose and complete therapy, may have improved outcomes compared with a person whose dose was reduced or discontinued owing to comorbidities or other risk factors for death.

Dose delays, dose reductions, and reduced RDI were also common among younger patients with NSCLC (i.e., 1849 years old); however, the reasons for this pattern in younger patients (e.g., pressure to continue to work, family duties, travel, adjustment to a new life-limiting diagnosis) may differ from the reasons in older patients (e.g., toxicity, comorbidity) [9], and reasons for dose delays in this study are unknown. The measures of chemotherapy intensity varied between the NSCLC subgroups, with dose delays, dose reductions, and reduced RDI being more frequent in patients with squamous cell carcinoma than adenocarcinoma. Patients with squamous cell carcinoma may have worse prognosis because of the increased likelihood of smoking, paraneoplastic syndrome, and ineligibility for many targeted therapies relative to patients with adenocarcinoma $[17,18]$.

Patterns for these measures of chemotherapy intensity also varied widely across chemotherapy regimens. Dose delays, dose reductions, and reduced RDI were more frequent in patients receiving treatment with carboplatin + gemcitabine or carboplatin + paclitaxel than the other chemotherapy regimens analyzed in this study. Pemetrexed + carboplatin had the highest mean RDI observed. Pemetrexed + bevacizumab + cisplatin had the lowest frequency of reduced RDI; pemetrexed + cisplatin had the lowest frequency of dose delays and dose reductions.

High rates of severe neutropenia (24.4\%) were observed. Primary prophylaxis with CSF, which can help maintain the chemotherapy dose intensity [9], was received by $18.3 \%$ of patients, which may have affected mortality in this patient population.

Our results demonstrating the relationship between RDI $<85 \%$ and an increased risk of death in the multivariable analysis are consistent with results from an earlier study that found significantly reduced OS (7 versus 10 months) in patients with advanced NSCLC who received RDI $<80 \%$ versus RDI $>80 \%[12]$. They are also similar to those from another study in patients with advanced epithelial ovarian cancer treated with platinum-based chemotherapy in which delivered RDI $<85 \%$ was associated with significantly reduced OS compared with delivered RDI $>85 \%$ [19]. In contrast, another study reported no difference in OS for patients with advanced NSCLC stratified by RDI $<90 \%$ versus $\geq$ $90 \%$, which may be due to differences in the RDI thresholds ( $90 \%$ versus $85 \%$ in the present study), type of analysis (landmark analysis versus log-rank, respectively), and minimum number of cycles of chemotherapy received for inclusion in the analysis (1 versus 4) [20].

Compared with the present study, an earlier study conducted in patients receiving adjuvant or neoadjuvant chemotherapy for stage 1-3A NSCLC found higher rates of RDI $<85 \%$ ( $79.3 \%$ versus $53.4 \%$ in the present study), dose delay $\geq 7$ days (63.9\% versus $39.1 \%$ ), and dose reductions $\geq 15 \%$ (83.6\% versus $60.5 \%$ ) for carboplatin plus paclitaxel [6]. These results suggest that physicians are more likely to decrease dose intensity in patients with less severe disease, hence confounding the results of this retrospective study.

Limitations of this study included the potential for selection bias due to the retrospective design and the possibility that inadequate or inaccurate codes could have introduced misclassification bias. Additionally, any services, medications, and procedures provided outside the McKesson Specialty Health/US Oncology Network were not captured by the iKnowMed EHR database and could not be ascertained in the study. We also did not have documentation regarding reasons for dose delays or reductions. This study relied on coded data fields in the iKnowMed database rather than on data extracted from medical charts, limiting our ability to determine certain clinical characteristics (e.g., histopathologic classification, comorbidities) and understand reasons for dose delays and reductions. There was no control for comorbid illness, which limited the ability to determine whether higher RDI was reflective of a healthier patient population and whether comorbid illness confounded CSF use and OS. The treatment patterns and clinical outcomes reflect the specific study time frame (2007-2010); extrapolation to current treatments is limited by changes in therapy since that time.

In summary, multivariable analysis in this study suggested that dose delay $\geq 7$ days was significantly associated with a decreased risk of death, whereas RDI $<85 \%$ was significantly associated with an increased risk of death. The finding that dose reduction alone was not a significant predictor of survival warrants further investigation. These results suggest that different measurements of dose variation may have different sensitivity in predicting prognosis. Understanding the complex effect of dose intensity on outcomes will be important for managing toxicities and improving survival; research studies such as this one can inform decisions about supportive care, specifically CSF use, in patients with metastatic cancer. Further studies should investigate reasons for dose delays and 
reductions and also consider other potential confounders, including comorbid illness and socioeconomic factors.

Acknowledgments The authors also acknowledge Janet Espirito (McKesson Specialty Health, The Woodlands, TX) for data analysis.

Funding information This study was funded by Amgen Inc. Medical writing support was provided by Miranda Tradewell and James Balwit (Complete Healthcare Communications, LLC, North Wales, PA), whose work was funded by Amgen Inc.

\section{Compliance with ethical standards}

Conflict of interest McKesson Specialty Health received research funding from Amgen Inc. to complete this work. JC and GHL are principal investigators on research grants from Amgen Inc. for their respective institutions. ND and XJ are or were employees of McKesson Specialty Health. PKM, JG, and RB are or were employees of and own stock in Amgen Inc. DP has nothing to disclose.

Data sharing There is a plan to share data. This may include deidentified individual patient data for variables necessary to address the specific research question in an approved data-sharing request, also related data dictionaries, study protocol, statistical analysis plan, informed consent form, and/or clinical study report. Data-sharing requests relating to data in this manuscript will be considered after the publication date and (1) this product and indication (or other new use) have been granted marketing authorization in both the USA and Europe, or (2) clinical development discontinues and the data will not be submitted to regulatory authorities. There is no end date for eligibility to submit a data-sharing request for these data. Qualified researchers may submit a request containing the research objectives, the Amgen product(s) and Amgen study/ studies in scope, endpoints/outcomes of interest, statistical analysis plan, data requirements, publication plan, and qualifications of the researcher(s). In general, Amgen does not grant external requests for individual patient data for the purpose of re-evaluating safety and efficacy issues already addressed in the product labeling. A committee of internal advisors reviews requests. If not approved, requests may be further arbitrated by a Data Sharing Independent Review Panel. Requests that pose a potential conflict of interest or an actual or potential competitive risk may be declined at Amgen's sole discretion and without further arbitration. Upon approval, information necessary to address the research question will be provided under the terms of a data-sharing agreement. This may include anonymized individual patient data and/or available supporting documents, containing fragments of analysis code where provided in analysis specifications. Further details are available at the following: https://www.amgen.com/science/clinical-trials/clinical-datatransparency-practices/

Open Access This article is distributed under the terms of the Creative Commons Attribution 4.0 International License (http:// creativecommons.org/licenses/by/4.0/), which permits unrestricted use, distribution, and reproduction in any medium, provided you give appropriate credit to the original author(s) and the source, provide a link to the Creative Commons license, and indicate if changes were made.

\section{References}

1. National Cancer Institute (2017) Statistics at a glance: lung and bronchus. Available at: https://seer.cancer.gov/statfacts/html/ lungb.html. Accessed May 11, 2017
2. Kaniski F, Enewold L, Thomas A, Malik S, Stevens JL, Harlan LC (2017) Temporal patterns of care and outcomes of non-small cell lung cancer patients in the United States diagnosed in 1996, 2005, and 2010. Lung Cancer 103:66-74

3. Scheff RJ, Schneider BJ (2013) Non-small-cell lung cancer: treatment of late stage disease: chemotherapeutics and new frontiers. Semin Interv Radiol 30:191-198

4. National Comprehensive Cancer Network (2017) NCCN Clinical Practice Guidelines in Oncology (NCCN Guidelines $\left.{ }^{\circledR}\right)$ Non-small cell lung cancer, version 4.2017. Available at: https://www.nccn. org/professionals/physician_gls/pdf/nscl.pdf. Accessed January 23, 2017

5. Sheehan RG, Balaban EP, Frenkel EP (1993) The impact of dose intensity of standard chemotherapy regimens in extensive stage small cell lung cancer. Am J Clin Oncol 16:250-255

6. Denduluri N, Patt DA, Wang Y, Bhor M, Li X, Favret AM, Morrow PK, Barron RL, Asmar L, Saravanan S, Li Y, Garcia J, Lyman GH (2015) Dose delays, dose reductions, and relative dose intensity in patients with cancer who received adjuvant or neoadjuvant chemotherapy in community oncology practices. J Natl Compr Cancer Netw 13:1383-1393

7. Dale DC, Bonilla MA, Davis MW, Nakanishi AM, Hammond WP, Kurtzberg J, Wang W, Jakubowski A, Winton E, Lalezari P et al (1993) A randomized controlled phase III trial of recombinant human granulocyte colony-stimulating factor (filgrastim) for treatment of severe chronic neutropenia. Blood 81:2496-2502

8. Douillard JY, Rosell R, De Lena M, Carpagnano F, Ramlau R, Gonzales-Larriba JL, Grodzki T, Pereira JR, Le Groumellec A, Lorusso V, Clary C, Torres AJ, Dahabreh J, Souquet PJ, Astudillo J, Fournel P, Artal-Cortes A, Jassem J, Koubkova L, His P, Riggi M, Hurteloup P (2006) Adjuvant vinorelbine plus cisplatin versus observation in patients with completely resected stage IB-IIIA nonsmall-cell lung cancer (Adjuvant Navelbine International Trialist Association [ANITA]): a randomised controlled trial. Lancet Oncol 7:719-727

9. Lyman GH (2009) Impact of chemotherapy dose intensity on cancer patient outcomes. J Natl Compr Cancer Netw 7:99-108

10. Culakova E, Thota R, Poniewierski MS, Kuderer NM, Wogu AF, Dale DC, Crawford J, Lyman GH (2014) Patterns of chemotherapyassociated toxicity and supportive care in US oncology practice: a nationwide prospective cohort study. Cancer Med 3:434- 444

11. Ramsden K, Laskin J, Ho C (2015) Adjuvant chemotherapy in resected stage II non-small cell lung cancer: evaluating the impact of dose intensity and time to treatment. Clin Oncol (R Coll Radiol) 27:394-400

12. Luciani A, Bertuzzi C, Ascione G, Di Gennaro E, Bozzoni S, Zonato S, Ferrari D, Foa P (2009) Dose intensity correlate with survival in elderly patients treated with chemotherapy for advanced non-small cell lung cancer. Lung Cancer 66:94-96

13. Denduluri N, Lyman GH, Wang Y, Morrow PK, Barron R, Patt D, Bhowmik D, Li X, Bhor M, Fox P, Dhanda R, Saravanan S, Jiao X, Garcia J, Crawford J (2018) Chemotherapy dose intensity and overall survival among patients with advanced breast or ovarian cancer. Clin Breast Cancer 18:380-386

14. National Cancer Institute (2010) Common Terminology Criteria for Adverse Events v4.03 (CTCAE). Available at: https://ctep.cancer. gov/protocolDevelopment/electronic_applications/ctc.htm. Accessed 31 May 2019

15. National Comprehensive Cancer Network (2012) NCCN Clinical Practice Guidelines in Oncology (NCCN Guidelines ${ }^{\circledR}$ ) myeloid growth factors, version 1.2012. Available at: http://www.nccn.org/ professionals/physician_gls/pdf/myeloid_growth.pdf. Accessed May 25, 2012

16. Havrilesky LJ, Reiner M, Morrow PK, Watson H, Crawford J (2015) A review of relative dose intensity and survival in patients with metastatic solid tumors. Crit Rev Oncol Hematol 93:203-210 
17. Kawase A, Yoshida J, Ishii G, Nakao M, Aokage K, Hishida T, Nishimura M, Nagai K (2012) Differences between squamous cell carcinoma and adenocarcinoma of the lung: are adenocarcinoma and squamous cell carcinoma prognostically equal? Jpn J Clin Oncol 42:189-195

18. Kanaji N, Watanabe N, Kita N, Bandoh S, Tadokoro A, Ishii T, Dobashi H, Matsunaga T (2014) Paraneoplastic syndromes associated with lung cancer. World J Clin Oncol 5:197-223

19. Hanna RK, Poniewierski MS, Laskey RA, Lopez MA, Shafer A, Van Le L, Crawford J, Dale DC, Gehrig PA, Secord AA, Havrilesky LJ, Lyman GH (2013) Predictors of reduced relative dose intensity and its relationship to mortality in women receiving multi-agent chemotherapy for epithelial ovarian cancer. Gynecol Oncol 129: 74-80

20. Brunetto AT, Carden CP, Myerson J, Faria AL, Ashley S, Popat S, O'Brien ME (2010) Modest reductions in dose intensity and druginduced neutropenia have no major impact on survival of patients with non-small cell lung cancer treated with platinum-doublet chemotherapy. J Thorac Oncol 5:1397-1403

Publisher's note Springer Nature remains neutral with regard to jurisdictional claims in published maps and institutional affiliations. 